\title{
Corela
}

Cognition, représentation, langage

HS-32 | 2020

Les postures énonciatives

\section{Dialogisme et points de vue en confrontation : comment appliquer les postures énonciatives à l'analyse des « détournements »?}

\section{Émilie Devriendt}

\author{
(2) OpenEdition \\ Journals \\ Édition électronique \\ URL : https://journals.openedition.org/corela/12081 \\ DOI : 10.4000/corela.12081 \\ ISSN : 1638-573X \\ Éditeur \\ Cercle linguistique du Centre et de l'Ouest - CerLICO
}

Référence électronique

Émilie Devriendt, « Dialogisme et points de vue en confrontation : comment appliquer les postures énonciatives à l'analyse des « détournements »? », Corela [En ligne], HS-32 | 2020, mis en ligne le 05 novembre 2020, consulté le 14 juillet 2021. URL : http://journals.openedition.org/corela/12081 ; DOI https://doi.org/10.4000/corela.12081

Ce document a été généré automatiquement le 14 juillet 2021.

\section{(c) (i) (2)(2)}

Corela - cognition, représentation, langage est mis à disposition selon les termes de la licence Creative Commons Attribution - Pas d'Utilisation Commerciale - Partage dans les Mêmes Conditions 4.0 International. 


\title{
Dialogisme et points de vue en confrontation : comment appliquer les postures énonciatives à l'analyse des « détournements »?
}

\author{
Émilie Devriendt
}

LA RÉVOLUTION

EST SÉRIEUSE MAIS

NE VOUS PRENEZ PAS

TROP AU SÉRIEUX

(un graffiti en Mai 1968)

\section{Introduction}

Mon intérêt pour les postures énonciatives est né en 2016, d'un besoin théorique et pratique : à l'occasion d'un colloque à Besançon intitulé Les acteurs du discours: de l'énonciateur à l'acteur social ${ }^{1}$, j'ai commencé à travailler sur un corpus constitué du texte de 394 inscriptions graffitées pendant l'occupation/libération de la Sorbonne en $1968^{2}$. Parmi ces énoncés se trouvaient notamment de très nombreux détournements ${ }^{3}$, bien décrits par ailleurs comme phénomènes dialogiques. L'argumentaire du colloque de Besançon m'incitait d'autant plus à creuser l'analyse de leur fonctionnement énonciatif, que leur énonciation «aphorisante» (Maingueneau 2012), jointe à l'anonymat de la plupart des énoncés graffités, semblait offrir des prises peu commodes à une analyse à même d'éclairer, au plan discursif, les pratiques des acteurs et actrices du mouvement des occupations de Mai (Viénet 1968). Pouvait-on décrire, et d'abord identifier, en termes énonciatifs, les nuances de l'accord, du désaccord, au sein de la communauté occupante même, comme vis-à-vis de ses extérieurs? Les postures énonciatives m'avaient paru fournir des outils pertinents - mais je ne crois pas l'avoir démontré par la mise en œuvre que j'en ai faite alors, conditionnée par la visée plus générale de mon étude. Le colloque de Toulon consacré aux propositions d'Alain 
Rabatel était pour moi l'occasion d'aborder de front ces questions à partir des cas de détournement attestés dans les graffitis de Mai, et c'est sur ce type d'énoncés dialogiques que j'ai choisi de centrer ma réflexion.

Après avoir situé et défini le procédé du détournement au sein de la problématique générale du dialogisme et de l'effacement énonciatif (partie 1), je m'attacherai à analyser des cas à l'aide des postures énonciatives, tout en interrogeant l'articulation des niveaux énonciatif et pragmatique (partie 2). La partie 3 me permettra d'approfondir les questions soulevées par ces analyses; j'y reviendrai à nouveaux frais sur la notion de " points de vue en confrontation » (Rabatel 2008a) dans l'énonciation des phénomènes dialogiques étudiés. Ce faisant, j'aborderai la question de l'articulation entre postures énonciatives et "positionnement sociologique " (Rabatel 2015a) et/ou enjeux (inter)actionnels et idéologiques (Rabatel 2004).

\section{Le détournement comme fait (et énoncé) dialogique}

3 Je m'intéresse ici aux travaux linguistiques qui ont explicitement travaillé sur des cas de détournement, sachant que ce phénomène a pu être analysé sous d'autres appellations, dans des cadres théoriques variés ${ }^{4}$.

\subsection{Définition énonciative du détournement}

4 Dans son étude de 2005 portant sur les titres de presse, Leroy se propose « de décrire le détournement (traité ailleurs en termes d'allusion, de stéréotypes et de clichés, de défigement...) comme un phénomène purement dialogique, en tâchant d'en caractériser les fonctionnements énonciatifs généraux, sans (trop) s'arrêter aux effets de sens ponctuels" (p. 202). L'auteure propose une première définition de ce phénomène $e^{5}$ :

(...) un procédé discursif consistant à produire un énoncé reprenant certaines marques linguistiques d'un autre énoncé, de façon à ce que celui-ci soit reconnaissable mais sans pour autant le reproduire à l'identique (...). On peut dire qu'avec le détournement, on "déforme " un énoncé pour en produire un autre, ressemblant mais pourtant différent.

Voici quelques exemples empruntés à mon corpus, où apparaissent en gras les opérations de modification du signifiant de $\mathrm{E}_{0}{ }^{6}$, ces opérations étant cumulables dans un même énoncé (substitution de termes homophones; substitution de termes sans similitude phonique nette ; ajout d'éléments à l'énoncé $\mathrm{E}_{0}$, jeu avec la négation, etc) :

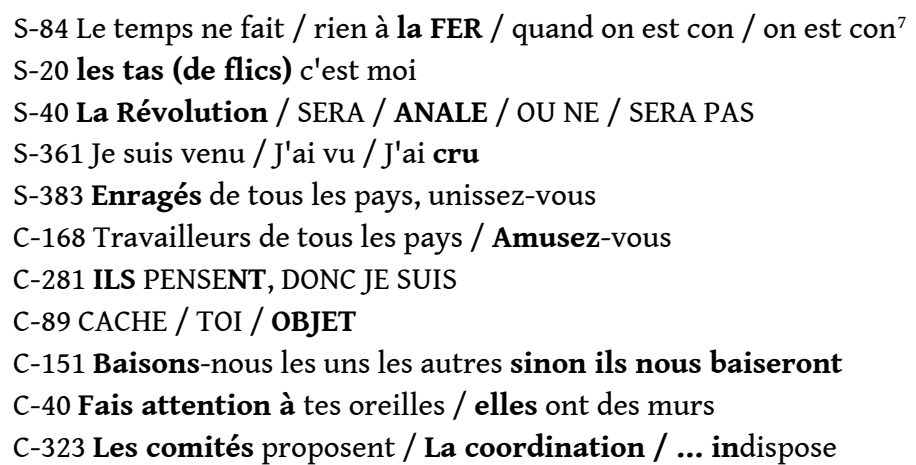


L'auteure s'interroge dans un second temps sur le statut « des cas, fréquents dans la presse, où l'énoncé détourné (...) est repris à l'identique, sans aucune modification formelle » (ibid.). Elle les analyse comme

(...) des cas particuliers de détournement, où l'écart se fait au niveau de la situation d'énonciation et non de la forme linguistique : si ces détournements reprennent un énoncé à l'identique, il y a décalage au niveau de la référence (...) et des conditions d'énonciation (...). Il s'agit donc d'un type de détournement sans marque formelle... (p. 211-212)

7 Voici quelques exemples empruntés à mon corpus :

C-230 ô TOI QUI / le SAVAIS

S-280 Toute destruction est créatrice

C-207 Puisque j'ai commencé de / rompre le silence, / Mâdame, il faut poursuivre.

S-302 Aimez-vous les uns les autres

8 Ces détournements «situationnels " posent des problèmes d'identification encore plus importants que les précédents, non seulement du fait de l'absence de marque d'opérations de modification de $\mathrm{E}_{0}$, mais aussi du fait du caractère «aphorisant » de l'énonciation graffitée - c'est-à-dire produisant des énoncés (nommés aphorisations) «(...) qui ne sont pas pri[s] dans la continuité d'un texte » (Maingueneau 2012:8). Je ne les évoquerai ici qu'à la marge.

\subsection{Le détournement comme marqueur et/ou comme fait de dialogisme}

9 La définition de Leroy (2005) s'inscrit explicitement dans la continuité des travaux de Bres, où le détournement est envisagé comme un marqueur de dialogisme, tout en problématisant cette approche ${ }^{8}$ par la mise en évidence d'une double dimension du phénomène étudié comme un "marqueur d'hétérogénéité montrée (explicitement marquée) ", « et en même temps comme un fait dialogique, relevant de l'hétérogénéité constitutive" (p. 212). De fait, Bres semble avoir modifié sa propre approche du phénomène en tenant compte des résultats de cette étude. Ainsi, dans Bres (2017b : 98), il en propose une définition, absente de Bres (2001) :

La figure du détournement consiste en la «superposition de deux (fragments d')énoncés » (Leroy 2005 : 203), comme si l'énoncé (E) s'inscrivait - syntaxiquement et/ou lexicalement - dans tout ou partie d'un énoncé (e) (...). Le plus souvent, le détournement ne se marque par aucun indice, et n'est interprétable comme tel que par notre mémoire discursive (Moirand 2003a) qui permet d'entendre, sous (E), un énoncé (e). Procédant de l'interaction avec un énoncé antérieur effectif, le dialogisme de détournement est toujours d'ordre interdiscursif ${ }^{9}$.

10 Cette insistance («le plus souvent») sur les cas d'absence d'« indices » est davantage explicitée dans Bres (2017a). Dans cet article, le détournement (ou «allusion», qui reprend la terminologie d'Authier-Revuz) est défini par les deux traits suivants: «effacement du marquage de l'interaction $[\mathrm{E}] /[\mathrm{e}]^{10}$ »; " présence sous-entendue [de l'interaction énonciative] inférable d'une trace parfois peu évidente lorsqu'elle n'est en rien signifiée, voire purement interprétative » (voir aussi Bres 2016 : 129). De cela, je retiens deux éléments concernant le phénomène étudié : la question de l'effacement énonciatif (qui concerne à la fois la source énonciative de l'énoncé rapporté/détourné et le marquage énonciatif du détournement); ce faisant, l'importance de la dimension interprétative (reposant sur la mémoire discursive de l'énonciataire et/ou les 
inférences qu'il peut produire au vu du co(n)texte de l'énoncé $\left.{ }^{11}\right)$, déjà bien soulignée dans Authier-Revuz (2000), Moirand (2007), Salvan (2010).

\subsection{Dialogisme et point de vue}

11 Si Leroy $(2005)$ et Bres $(2001 / 2017,2016)$ abordent le détournement comme phénomène strictement énonciatif, Bres (2016) le fait en discutant, notamment, avec Rabatel (2016), autour de la notion de point de vue (PDV). Il n'est pas dans mon propos ici d'entrer dans (le détail de) cette discussion. Leur opposition théorique sur l'approche du dialogisme - dont tous deux se revendiquent - va en revanche m'aider à situer ma démarche. Citons Bres $(2016: 123)$ :

Il nous semble (...) que pour rendre compte de l'hétérogénéité énonciative d'énoncés [dialogiques], il n'est pas besoin de faire appel à la notion de point de vue qui tente d'appréhender le sens en dehors des mots, le contenu libéré de sa forme. Il suffit d'analyser lesdits énoncés comme procédant d'une interaction avec un autre énoncé, interaction qui laisse des traces plus ou moins explicites.

Comme Bres, je travaille en analyse du discours et, pas plus que lui, je ne situe mes travaux dans le cadre théorique défini par Rabatel. La discussion à laquelle nous invitait le colloque de Toulon me conduira toutefois (a) à pratiquer une approche « empathique » de ses propositions, visant en particulier à utiliser sans la dénaturer sa conception du PDV - qui, à bien le lire, n'a rien d'«idéaliste » - et, accessoirement, du 《sujet $~^{12}$, (b) à chercher à comprendre ce que serait dans son cadre l'équivalent de ce que Bres nomme interaction dialogique (en référence à Bakhtine/Volochinov), et (c) à étudier des objets propres à favoriser les comparaisons : ainsi, je ne parlerai que des énoncés/PDV correspondant à un contenu propositionnel, intégrant une ou plusieurs propositions (voir Rabatel 2014: 93, n. 3). Ce faisant, j'entends défendre l'idée que l'approche en termes de PDV n'est pertinente, s'agissant à tout le moins des détournements de mon corpus, que sous certaines conditions que je m'efforcerai de caractériser.

\section{Le fonctionnement énonciativo-pragmatique du détournement au prisme de la topique énonciative de Rabatel}

13 Rabatel définit son approche générale des faits de dialogisme comme "énonciativopragmatique $»^{13}$ (impliquant les niveaux sémantique et référentiel), celle-ci ne se voulant pas coupée du niveau sociodiscursif ${ }^{14}$. Si la dimension énonciative et dialogique - les auteur.es parlent toutefois de polyphonie - est bien présente dans l'approche de Grésillon et Maingueneau (1984), le détournement y est envisagé comme un "procédé discursif » (p. 114), ce qui implique de surcroît, et de manière privilégiée, une attention portée au niveau sémantique et pragmatique des énoncés produits dans le cadre de cette « pratique » (ibid.).

\subsection{Captation / subversion, accord / désaccord, postures énonciatives}

Grésillon et Maingueneau définissent deux fonctions (ou visées, ou régimes interprétatifs) du détournement de proverbes ${ }^{15}$. Il me paraît important de distinguer 
dans cette définition deux dimensions: sémantique et pragmatique. Au niveau sémantique, la captation est décrite comme convergence sémantique entre $\mathrm{E}_{1}$ et $\mathrm{E}_{0}$ (elle " consiste à détourner en allant au maximum dans le sens de la structure sémantique ainsi exploitée ", p. 115), et la subversion comme " contradiction » sémantique entre $E_{1}$ et $\mathrm{E}_{0}$ (ibid.). Au niveau pragmatique, il s'agit, dans la captation, «d'utiliser à son profit l'autorité proverbiale » (p. 115), et dans la subversion, de « contester » cette autorité pour «ruiner les vérités établies». (p. 114). Au plan discursif et pragmatique, les auteures distinguent en outre deux « régimes » de mise en œuvre du détournement (p. 114) : un régime ludique (le « détournement entre dans le cadre des purs jeux de mots, et n'est pas au service d'un enjeu idéologique, politique ») et un régime militant (où le détournement « vise à donner de l'autorité à un énoncé ou à ruiner celle du proverbe au nom d'intérêts de divers ordres »), soulignant également que «(...) les subversions qui visent le signifié des proverbes attestés ont inévitablement une portée militante " (p. 119) ${ }^{16}$.

Il me semble possible de rapprocher le niveau sémantique de cette analyse des détournements en termes de captation et de subversion ${ }^{17}$, de ce que Rabatel associe quant à lui au niveau du point de vue ( $\left.\mathrm{PDV}^{18}\right)$, et plus particulièrement, à la question de la prise en charge ou non prise en charge du PDV - dans les cas de «disjonction présupposée des instances de prise en charge et de validation » ou " hétéro-PDV », c'est-à-dire des cas de dialogisme. Dans Rabatel (2012a), la véritable prise en charge d'un PDV, aussi définie comme "fusion hétérodialogique des instances de validation de E1 et e2 ou E1 $1^{19}$ ", est nommée accord, par opposition au désaccord ou "refus de la (con)fusion des instances de validation de E1 et e2 ou E1'» (p. 30), ces deux pôles étant neutralisés dans les cas de prise en compte seule (je reviendrai sur cette notion dans la partie 3 ).

Rabatel articule par ailleurs la question de la prise en charge des PDV à celle des positionnements énonciatifs possibles: par redoublement autodialogique ou par dédoublement hétérodialogique. Le redoublement n'est associé qu'au seul accord; en revanche, le dédoublement est applicable à toutes les étapes du continuum accorddésaccord. En relève aussi la "neutralité " associée à la prise en compte seule. Le niveau des postures énonciatives est articulé à ces deux premiers niveaux, et en particulier à celui des positionnements :

Quant aux postures énonciatives de co-, sur- et sous-énonciation, elles affinent la notion de dédoublement ou de redoublement énonciatifs au plan interactionnel en permettant de mieux baliser toutes les nuances de l'accord, installant la discordance dans la concordance (et inversement), en fonction des ajustements cognitifs et interactionnels qui se jouent au fil du discours. (p. 24)

Il est important de souligner que «[1]es postures correspondent aux relations entre énonciateurs dans la co-construction linguistique d'un "même" PDV, considérant que les formulations n'altèrent pas significativement le PDV initial» (p. 34). De fait, c'est cette co-construction qui semble avoir été l'objet privilégié des travaux de Rabatel consacrés aux postures, au détriment du pôle appelé « désaccord ${ }^{20}$.

De ce qui précède, et si l'on accepte d'assimiler accord et captation d'une part, désaccord et subversion d'autre part, sur une base sémantique et propositionnelle, on peut partir du principe :

19 - que les détournements "parasites ${ }^{21}$ sont susceptibles de relever, soit de la coénonciation, soit de la surénonciation, soit de la sousénonciation; 
- que les détournements « subversifs » sont susceptibles de relever de l'énonciation de deux PDV alternatifs, opposés ou contradictoires, impliquant un même objet sur lequel porterait cette opposition. Comme tels, ils ne relèveraient pas de la problématique des postures énonciatives ${ }^{22}$, sauf dans les cas où la subversion ne viserait pas "le contenu du proverbe $E_{0}$ " mais «dénonce[rait] celui qui a substitué à un $E_{0}$ jugé positif un $E_{1}$ inadmissible» (Grésillon et Maingueneau art. cit. : 120). Dans les termes de Rabatel, les cas de détournements subversifs à interprétation ironique pourraient être analysés en termes de surénonciation (un seul PDV contre 2 PDV), à condition toutefois qu'ils ne soient pas antiphrastiques (voir Rabatel $2013: 39$ sq.).

\subsection{Illustrations}

Je propose de tester la validité du rapprochement proposé précédemment, en examinant quelques exemples.

\subsubsection{Cas de captation}

Si l'on s'en tient au critère général de la "convergence sémantique entre les deux énoncés, $E_{0}$ et $E_{1}$ » définissant, selon Grésillon et Maingueneau (p. 117-118), la captation, on aurait affaire ici à un cas de coénonciation :

S-84 Le temps ne fait / rien à la FER / quand on est con / on est con

En d'autres termes, le contenu propositionnel ( $\mathrm{CP}$ ) de l'énoncé détourné $\mathrm{E}_{0}$ est pris en charge. Le détournement repose sur une opération de substitution de segments homophones assurant la superposition des deux énoncés. $\mathrm{E}_{0}$ (Le temps ne fait rien à l'affaire), extrait du texte éponyme du chanteur libertaire Georges Brassens, est ainsi capté sémantiquement et pragmatiquement pour viser une cible habituelle des graffiteurs de la Sorbonne: la Fédération des Etudiants Révolutionnaires, une organisation trotskiste. Cet exemple de coénonciation est proche de ce que Maingueneau $(2004,2012)$ nomme "particitation $»^{23}$. Mais, le plus souvent, les détournements « parasites » marquent des nuances dans l'accord :

S-383 Enragés de tous les pays, unissez-vous

C-168 Travailleurs de tous les pays / Amusez-vous

Dans ces deux exemples, le détournement de l'énoncé attribuable au Manifeste du parti communiste passe par une substitution : celle du $\mathrm{N}$ appellatif (Enragés pour travailleurs) en S-383; celle du verbe (amuser pour unir en $\mathrm{C}-168^{24}$ ). Dans le premier cas, le $\mathrm{CP}_{0}$ est inchangé, mais la substitution nominale ${ }^{25}$ semble constituer une mise à jour de l'énoncé de Marx et Engels en référence à la situation révolutionnaire - si ce choix lexical paraît "déprolétariser » la catégorie initiale, il renvoie sans doute plutôt à la redéfinition situationniste du prolétaire comme individu dépossédé de sa vie. On a ici un cas de sousénonciation. Dans le second, $\mathrm{CP}_{0}$ est sensiblement altéré. Dans la mesure où union de classe et amusement ne sont pas contradictoires - selon une inscription, c'est l'ennui qui est contre-révolutionnaire ${ }^{26}$ - et encore moins incompatibles (la "joie » d'être ensemble est souvent mentionnée par les protagonistes de Mai), cette réorientation du prédicat de $\mathrm{CP}_{0}$ me fait conclure à un cas de surénonciation plutôt qu'à un désaccord. 


\subsubsection{Cas de subversion (ironique)} tels :

S-20 les tas (de flics) c'est moi

C-281 ILS PENSENT, DONC JE SUIS

Comme dans S-84, on a affaire avec S-20 à un détournement par substitution homophonique (les tas / l'État), mais celle-ci est associée à un ajout «subversif» qui, plutôt que de substituer un référent à un autre, contribue à présenter le référent initial sous un angle différent: $\mathrm{PDV}_{1}$ ne contredit par $\mathrm{PDV}_{0}$, il le complète pour faire apparaitre la réalité répressive de l'État : il s'agit donc bien d'un cas de surénonciation. S'il est ironique, c'est que PDV ${ }_{1}$ 'est pas celui de L1, mais d'un pseudo-L1, qu'on pourrait noter 12'/e2', en tant qu'il prendrait en charge, en le surénonçant, $\mathrm{PDV}_{0}$. Ce 12'/e2' pourrait valoir pour tout individu représentant le pouvoir étatique (par exemple, Maurice Grimaud, le préfet de police de la ville de Paris, ou De Gaulle luimême, par analogie avec 12/e2, à savoir Louis XIV, source énonciative souvent attribuée à $\mathrm{E}_{0}$ ). S'agissant de $\mathrm{L} 1 / \mathrm{E} 1$, on peut donc parler de prise en charge "prétendue " (ironique), son effet subversif consistant à railler l'autorité du pouvoir tout en la démystifiant.

En C-281 il y a substitution de ils à je, ce qui aboutit à une dissociation entre pensée (conscience) et existence, pour signifier une individualité (ou une conscience) fondamentalement aliénée. Dans cette logique, on peut également lire une syllepse de sens sur suis ("être» ou «suivre»). La subversion vient d'une part de cette transformation de $\mathrm{CP}_{0}$, et d'autre part de l'énonciation rapportée, présentée comme directe (je), alors qu'il faut là encore l'associer à l2'/e2', le fonctionnement énonciatif de $\mathrm{E}_{1}$ étant proche d'un discours direct libre qui ferait parler un représentant de la « classe » des individus aliénés. Il y a ainsi dénonciation ironique du $\mathrm{CP}_{1}$ (en référence à l'aliénation), donc ( $\left.\mathrm{du} \mathrm{PDV}_{1}\right)$ de l'énonciateur susceptible de le prendre en charge (e2') en l'occurrence, de tout individu en proie à la « fausse conscience ».

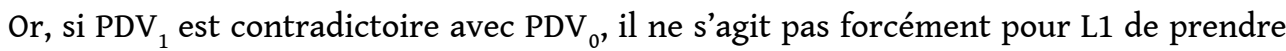
en charge la "vérité respectable » attribuée à $\mathrm{E}_{0}$, contrairement aux cas examinés par Grésillon et Maingueneau (p. 118-119) ${ }^{27}$. Le détournement subversif impliquerait donc ici à la fois un refus de prise en charge de PDV ${ }_{1}$ et une simple prise en compte, ou une prise en charge, éventuelle, de $\mathrm{PDV}_{2}$. L'analyse en termes de postures n'est donc susceptible de fonctionner que dans cette dernière éventualité et l'on aurait alors surénonciation de l'énoncé cartésien $\mathrm{E}_{0}$. Au niveau pragmatique, cette lecture ne paraît pas la plus satisfaisante, la subversion visant vraisemblablement un énonciataire directement impliqué dans les évènements en cours. En d'autres termes, la confrontation des PDV ne semble véritablement pertinente sur le plan discursif qu'au niveau du dédoublement énonciatif entre L1 et l2'/e2' : elle n'implique manifestement pas 12/e2 (ni Descartes, ni ses aphoriseurs potentiels).

\section{Cache-toi objet? Le PDV en question}

De fait, la question de la pertinence des postures me paraît liée à cette identification des conditions de la «confrontation» des PDV postulée par Rabatel. Si je crois qu'on 
peut voir dans l'idée de «PDV en confrontation» l'équivalent chez Rabatel de l'« interaction dialogique» chez Bres, la notion de PDV, du fait de sa dimension sémantique, complexifie l'approche du dialogisme. En effet, dans le cadre énonciatif «radical » de Bres, identifier l'allusion suffirait à rendre compte de l'hétérogénéité énonciative (voir 1.3). Chez Rabatel en revanche, l'énoncé étant inséparable d'un PDV (fût-il embryonnaire), son interaction avec d'autres énoncés implique en outre qu'un "même » objet soit envisagé28 - sans quoi les notions d'accord, désaccord ou de neutralité (et donc de prise en charge comme de prise en compte) n'auraient pas grand sens.

\subsection{PDV en confrontation et « objet » commun : quelle prise en compte?}

La question de l'objet doit en premier lieu être abordée au niveau sémantique et référentiel. S'agissant de la référence, Leroy (2005) a déjà bien montré que tout détournement implique une dé- et une re-contextualisation de $\mathrm{E}_{0}$ (voir aussi Maingueneau 2012), en particulier dans les cas de référenciation déictique : en C-281, je n'est pas un sujet philosophique, vous désigne les lecteurs potentiels de S-383 et C-168, on désigne notamment la FER en S-84. S'agissant du plan sémantique, on peut chercher à comparer les objets ou $\mathrm{CP}$ respectifs de $\mathrm{E}_{0}$ et $\mathrm{E}_{1}$, en s'appuyant sur la structure des propositions (sujet et prédicat), et ce dans la mesure où les PDV sont « reconstructibles à partir des traces de référenciation des objets » (Rabatel 2014 : 92). Ainsi, les exemples analysés dans la partie 2 correspondent de manière plus ou moins prototypique à deux cas de figure : soit la proposition est reprise « à l'identique » (sujet et prédicat), c'est-àdire en fait, moyennant des variantes, graduelles, n'altérant pas radicalement $\mathrm{CP}_{0}(\mathrm{~S}-84$, S-383 ${ }^{29}$; soit la proposition est partiellement transformée, du fait de la modification du sujet (c'est le cas avec l'ajout dans $\mathrm{S}-20$ et la substitution de ils à je dans la première proposition de C-281) ou du prédicat (C-168, seconde proposition si l'on considère la syllepse «être» vs «suivre $\left.{ }^{30}\right)$, altérant $\mathrm{CP}_{0}$, sans que l'objet puisse être dit radicalement autre (là encore, il y a gradation). On voit au passage que le premier cas regroupe des exemples de coénonciation et de sousénonciation, le second des exemples de surénonciation.

31 J'envisage à présent un cas où le détournement transforme radicalement $\mathrm{CP}_{0}$, au point qu'il ne semble plus possible de parler d'un objet commun :

S-40 La Révolution / SERA / ANALE / OU NE / SERA PAS

$\mathrm{E}_{0}$ a pour source énonciative la plus probable le poète surréaliste André Breton ( $\mathrm{La}$ beauté sera convulsive ou ne sera pas), par ailleurs (parti)cité sur les murs en Mai. Ici, le sujet et le prédicat substitués sont a priori sans relation lexico-sémantique avec $\mathrm{CP}_{0}$ (même s'il serait toujours possible, dans une perspective " exégétique ", d'en trouver une). Il me semble que, dans ce cas, il n'est pas possible de dire que PDV ${ }_{0}$ est pris en compte dans l'énonciation de son détournement (et donc, qu'il n'y a ni neutralité, ni accord, ni désaccord). Dit autrement, il n'y aurait pas ici confrontation de PDV entre $E_{1}$ et $\mathrm{E}_{0}$, et ce malgré les traces de dialogisme repérables dans $\mathrm{E}_{1}{ }^{31}$. Sur le plan sémantique, il serait donc aussi difficile de parler de subversion ou de captation. Au plan pragmatique en revanche, si l'on tient compte de la présence d'inscriptions (d'inspiration) surréaliste(s) en Mai (dont $\mathrm{E}_{0}$ ), on pourrait voir dans l'allusion un « hommage » indirect à la formule comme à son auteur, et donc un cas de captation. On 
ne peut cependant évacuer l'hypothèse d'une subversion de l'énonciation prenant appui sur l'identité de 12/e2, précisément en tant qu' «auteur" (voir C-254 "Les écrivains sont des cons »), c'est-à-dire figure d'autorité ${ }^{32}$.

Plutôt que d'en conclure à la non-pertinence d'une confrontation de PDV dans des énoncés dialogiques tel S- 40 , ce qui pourrait sembler contre-intuitif vu l'inextricabilité entre énoncé et PDV chez Rabatel, et pour rester « empathique » avec son approche, il me semble plus «co-constructif» d'élargir la notion de prise en compte tout en spécifiant ce qu'on peut entendre par « objet ", au-delà de la dimension sémantique ou propositionnelle. Cette prise en compte élargie reviendrait dès lors à acter que quelque chose a été dit (écrit) sur quelque chose par quelqu'un (dans la perspective de Rabatel, il y a donc bien PDV), mais en "ciblant" (visant) l'un et/ou l'autre de ces trois paramètres de l'interaction (de la confrontation) dialogique : le $\mathrm{CP}$ de $\mathrm{E}_{0}$, et/ou ses conditions d'énonciation, et/ou plus spécifiquement sa source énonciative (12/e2). Les deux sections qui suivent visent à illustrer cette hypothèse, et impliquent une articulation graduelle des niveaux sémantique, énonciatif, pragmatique et discursif.

\subsection{Prise en compte visant les conditions génériques de l'énonciation : $E_{0}$ comme prêt-à-écrire}

Mon hypothèse est donc qu'il $\mathrm{y}$ a toujours prise en compte de $\mathrm{E}_{0}$ au sens défini précédemment. Reste alors à en déterminer les modalités, dont je postule qu'elles dépendent de plusieurs paramètres. Nous avons évoqué le $\mathrm{CP}$ en 3.1. J'envisage ici les conditions d'énonciation ${ }^{33}$, paramètre qui relève principalement du niveau énonciativo-pragmatique, articulé au niveau sémantique qu'il y ait ou non objet propositionnel partagé entre $E_{1}$ et $E_{0}$.

Il se trouve dans mon corpus un énoncé détournant la phrase célèbre de César ${ }^{34}$ :

S-361 Je suis venu / J'ai vu / J'ai cru

Le détournement repose sur la substitution d'un seul élément : le verbe de la troisième proposition. Ce verbe permet de conserver l'auxiliaire ainsi que la finale vocalique $\mathrm{du}$ participe passé. Au plan sémantique, vaincre est remplacé par croire, lui aussi en emploi absolu. On peut gager qu'il ne s'agit pas de croyance religieuse au sens strict, mais appliquée à un autre objet en lien avec la situation, comme par exemple dans S-35 : « Au sein de nos contradictions présentes / règne une foi insurrectionnelle / que nul n'arrêtera ». On pourrait donc associer la " croyance » à l'idée que la révolution était possible, conformément aux témoignages des protagonistes de Mai. Elle serait ici associée à (ou provoquée par) la découverte de la Sorbonne libérée. Dans une interaction syntaxico-sémantique entre $\mathrm{E}_{0}$ et $\mathrm{E}_{1}$, la « victoire » serait restituable comme complément effacé du verbe croire (croire que le renversement du vieux monde est possible). Vu la reprise de deux propositions à l'identique (impliquant seulement un changement de référent), et la substitution sémantiquement « compatible » du prédicat de la troisième, on a bien ici prise en compte et prise en charge de $\mathrm{PDV}_{0}$, et $\mathrm{E}_{1}$ serait donc analysable en termes de postures. Outre une lecture "sérieuse " (co-énonciation de $\mathrm{PDV}_{0}$ ), deux lectures sont possibles: sous-énonciation humoristique (autodérision quant à l'exaltation signifiée), ou sur-énonciation (auto-)ironique de PDV $_{1}$ (raillant la vanité d'une croyance naïve, opposée au constat d'échec du mouvement ou, à tout le moins, au constat d'un « retour à la normale ») ${ }^{35}$. 

fonctionnement dialogique de S-361 : si, comme on l'a vu, $\mathrm{PDV}_{0}$ est possiblement pris en charge, donc pris en compte au niveau sémantique, il semble bien davantage que ce soit ses conditions d'énonciation qui constituent l'objet visé dans l'interaction dialogique et, plus particulièrement, ses conditions formelles - indépendamment de 12/e2, qui pourrait même n'être plus être identifié en tant que César, sans que le fonctionnement dialogique s'en trouve modifié. Le style solennel associé ou non aux "actions d'éclat " $\mathrm{du}$ «discours guerrier» de $\mathrm{E}_{0}$ (Rabatel $2013:$ 38) serait ici, sur la seule base de son énonciation, capté (au plan pragmatique), et comme tel applicable à n'importe quelle situation, avec des effets divers. On a bien affaire à un "prêt à (ré)emploi » qui fait office de prêt-à-écrire, fonction de toutes les "aphorisations", en particulier des phrases célèbres (voir Maingueneau 2012, Ducard 2015) ${ }^{36}$.

On peut à présent associer S-40 (étudié en 3.1) à ce type de fonctionnement - et ce d'autant plus que la prise en compte de $\mathrm{CP}_{0}$ n'y est pas pertinente. Au plan pragmatique, c'est donc la structure formelle de leur énoncé voire, à travers elle, une autorité énonciative (celle de Breton) qui y serait soit subvertie, soit captée. Des cas de dissociation des paramètres de l'interaction dialogique se retrouvent aussi dans les détournements proverbiaux (voir par exemple C-323 «Les comités proposent / La coordination / ... indispose »), où le paramètre sémantique n'est pas pertinent dans la prise en compte (au sens large) de $\mathrm{E}_{0}$, qui cible alors exclusivement les conditions génériques de l'énonciation - et ce d'autant plus que sa source énonciative (paramètre 3) est indéterminée ${ }^{37}$.

Mon hypothèse est qu'il n'en va pas de même lorsque $E_{0}$ présente des enjeux idéologiques/politiques pertinents au vu de la situation d'énonciation de $E_{1}$ - et des intérêts actionnels du graffiteur. Or la prise en compte de ces enjeux dans l'analyse relève pour une grande part de l'interprétation, appuyée sur des éléments contextuels ${ }^{38}$ - donc d'une approche résolument pragmatique et discursive.

\subsection{Prise en compte visant la source énonciative de $E_{0}$ comme être du monde : PDV en confrontation et positionnement social}

Dans l'exemple précédent, j'ai analysé un cas où l'objet de la prise en compte n'est pas tant $\mathrm{CP}_{0}$, bien que cette analyse reste pertinente au niveau strictement sémantique et énonciatif, que les conditions formelles de son énonciation (son "style", si l'on veut). J'examine pour finir des cas où la prise en compte vise aussi, ou davantage, voire parfois exclusivement, le locuteur-énonciateur de $\mathrm{E}_{0}$. Contrairement à 3.2, il ne s'agit pas d'envisager 12/e2 comme pure instance énonciative, mais aussi comme «être du monde ", donc acteur social, ce qu'on pourrait appeler un locuteur-acteur. L'idée est de voir comment les postures énonciatives s'articulent aux positionnements sociaux - aux intérêts des locuteurs/acteurs et ce faisant aux enjeux de leurs interactions reprécisons donc que ce niveau d'analyse n'annule pas les précédents, mais s'articule à eux. Pour ce faire, j'envisagerai ici des cas d'interactions graffitées ${ }^{39}$, où le geste d'écriture peut signifier une intention de participer à la construction d'un PDV ou, au contraire, de contester les PDV signifiés sur les murs - et, ce faisant, un « marquage de territoire » en tant qu'acteur du mouvement des occupations. Le détournement s'y fait in praesentia, ce qui règle en quelque sorte la question de son identification ${ }^{40}$. 
Soit ces deux énoncés adjacents, sur les murs de la salle 308 à Censier :

C-285 Professeurs! / vous nous faites vieillir.

C-286 Elèves! Vous nous / maintenez jeunes

$\mathrm{C}-285$ est la matrice $\mathrm{E}_{0}$ (on compte plusieurs occurrences dans le corpus, en particulier dans la Sorbonne), la source énonciative étant le groupe des Enragés / Situationnistes. C-286 interagit avec lui par détournement. Ici PDV ${ }_{0}$ est pris en compte au niveau du CP, comme l'indique l'analyse sémantique des substitutions lexicales antonymiques: l'une complémentaire (élèves), les deux autres oppositives (jeunes; et maintenir s'opposant aspectuellement à la périphrase factitive). S'il y a bien opposition sémantique (donc subversion), on ne peut pas dire pour autant que $\mathrm{PDV}_{1}$ conteste $\mathrm{PDV}_{0}$, dans la mesure où la complémentarité des PDV signifiée dans l'adresse implique un positionnement sur un objet différent ${ }^{41}$. Il serait donc abusif de parler de désaccord - ou du reste d'accord. La prise en compte semble ainsi principalement viser 12/e2 (locuteur-énonciateur de C-285), qui n'est pas forcément un membre des Enragés, mais tout aphoriseur potentiel de $\mathrm{E}_{0}$, en tant que catégorisable comme « élève » (ou étudiant ?). La prise en compte de $\mathrm{E}_{0}$ vise donc 12/e2 dans la mesure où, au plan sociodiscursif, ce(s) locuteur(s)-acteur(s) sont aussi les énonciataires de $\mathrm{E}_{1}^{42}$. C-285 révèle les tensions ou contradictions présentes au sein du groupe des occupante-s de Censier, et l'enjeu stratégique du positionnement analysable dans C-286 doit être interprété au regard desdites contradictions. On pourrait parler ici de captation au plan pragmatique, dont le but pourrait être de renverser un rapport social existant (signifié dans $\mathrm{E}_{0}$ ) et ce faisant, de le... subvertir.

Dans les exemples suivants, le dialogisme est «traçable» dans l'enceinte de la Sorbonne :

S-313 La culture / est / l'inversion / de la vie (Grand amphithéâtre, porte E)

S-369 The university is the inversion of life (Escalier A, Entrée de l'Inspection d'académie)

S-82 La mysoginie est l'inversion de la vie...rilité (Galerie Robert Sorbon, salle dite de «Ronéo»)

S-313 est la matrice $E_{0}$, là encore attribuable au groupe des Enragés-Situationnistes ${ }^{43}$. $\mathrm{S}$-369 substitue un $\mathrm{N}$ dialoguant avec $\mathrm{E}_{0}$ par méronymie, comme le confirme l'association récurrente dans le corpus de culture et de professeurs : on a ici un cas de coénonciation. Le détournement est plus opaque en S-82: la première substitution (misogynie) est allotopique, bien qu'elle puisse être inférée d'une catégorisation de la culture ou de l'université comme institutions patriarcales (relation méronymique). La seconde est également allotopique, mais l'interaction s'y effectue sur la base de l'homographie (vie) qui, tout en renforçant la référence patriarcale (virilité), relie $\mathrm{CP}_{0}$ et $\mathrm{CP}_{1}$ au niveau du prédicat. Comme pour $\mathrm{C}-286$, la comparaison des $\mathrm{CP}$ pose la question du ou des paramètre(s) ciblé(s) dans la prise en compte (au sens large) de $\mathrm{E}_{0}$ (ici, S-313) : la dimension critique (pro)féministe introduite par $\mathrm{E}_{1}$ interroge sur la prise en charge potentielle, la «neutralité » ou le refus de prise en charge de $\mathrm{PDV}_{0}$. Mon analyse est qu'on parlera d'accord et de sur-énonciation si la cible du détournement est le patriarcat (et ses instanciations institutionnelles), mais de désaccord si la cible est le graffiteur de $\mathrm{E}_{0}$ en tant qu'acteur du mouvement : soit comme pro-Situationniste, soit comme révolutionnaire masculin ${ }^{44}$. Cette ambiguïté nous renvoie ici encore à la pertinence d'une articulation des approches énonciativo-pragmatique et discursive, voire sociologique et historique - comme aux limites même de ces approches confrontées à l'énonciation « aphorisante » graffitée. 


\section{Synthèse}

Les détournements, en tant qu'énoncés dialogiques, sont analysables dans l'optique de Rabatel - associant étroitement énonciation et référenciation - comme des phénomènes de points de vue en confrontation, qu'ils soient cumulatifs ou substitutifs. Cette analyse ne peut toutefois être menée, d'après moi, que sous certaines conditions. continuum accord-désaccord), la confrontation des PDV implique de pouvoir restituer un objet sémantique ou propositionnel commun à $\mathrm{E}_{0}$ et $\mathrm{E}_{1}$. Dans la mesure où un tel objet n'est pas toujours restituable, mais où l'interaction dialogique entre $E_{1}$ et $E_{0}$ n'en est pas moins identifiable ${ }^{45}$, en tant que définitoire du détournement, je propose d'élargir la notion de "prise en compte", tout en spécifiant la notion d' "objet» en trois paramètres pouvant servir de support d'interaction (ou confrontation) dialogique.

Dans cette perspective, la prise en compte de $\mathrm{PDV}_{0}$, entendue au sens large, n'implique pas forcément un objet sémantique (ou propositionnel) commun. Le détournement, s'il peut prendre pour objet (viser) le $\mathrm{CP}$ de $\mathrm{E}_{0}$ (paramètre 1), peut aussi viser ses conditions génériques d'énonciation, en particulier formelles (paramètre 2), et/ou encore, plus spécifiquement, la source énonciative de l'énoncé détourné, en tant qu'instance énonciative (paramètre 2) ou en tant qu'être du monde, locuteur-acteur (paramètre 3 ).

Au niveau pragmatique, L1/E1 peut donc se positionner avec (captation) ou contre (subversion) le $\mathrm{CP}_{0}$ (il peut dès lors y avoir accord, désaccord, ou "neutralité »), et/ou avec ou contre l'énonciation de $\mathrm{E}_{0}$, en visant plus ou moins, ou ne visant pas, les paramètres 2 et/ou 3. Dans les cas où les énoncés sont porteurs d'enjeux idéologiques, sociopolitiques ou (inter)actionnels marqués, cette visée peut servir une stratégie de positionnement, non seulement énonciativo-pragmatique, mais aussi "sociologique » (Rabatel 2015a) et "politique». L'analyse du dialogisme des détournements implique donc bien de dissocier, mais aussi de chercher à articuler, les niveaux sémantique, énonciatif, pragmatique et sociodiscursif.

Il reste que, quel(s) que soi(en)t le(s) niveau(x) d'analyse envisagé(s), les détournements, en particulier les détournements " aphorisés ", comme c'est le cas des énoncés graffités en mai 68 que j'ai étudiés ici, me semblent illustrer toute la complexité des faits de dialogisme - ou, plus largement, de la dialectique sociolangagière du même et de l'autre. Les propositions théoriques d'Alain Rabatel me paraissent contribuer non seulement à mieux comprendre et décrire les fonctionnements de cette dialectique, mais aussi à l'illustrer, par les réflexions et discussions qu'elles suscitent. Leur visée émancipatrice confirme, sur un autre plan, l'empathie - et la sympathie - qu'on peut avoir pour elles. 


\section{BIBLIOGRAPHIE}

ARON Paul, 2009, « Le pastiche comme objet d'étude littéraire. Quelques réflexions sur l'histoire du genre ", Modèles linguistiques, 60, 11-27.

AUTHIER-REVUZ Jacqueline, 1995/2012, Ces mots qui ne vont pas de soi. Boucles réflexives et noncoïncidences $d u$ dire, Limoges, Lambert Lucas.

AUTHIER-REVUZ Jacqueline, 2000, « Aux risques de l'allusion », in Murat M. (éd.), L'allusion dans la littérature, Paris, Presses de l'Université de Paris-Sorbonne, 209-235.

BRES Jacques, 2017b, « Dialogisme (marqueurs de) », in Détrie C., Siblot P., Verine B. et Steuckardt A. (éds), Termes et concepts pour l'analyse du discours. Une approche praxématique, Paris, H. Champion. BRES Jacques, 2017a, « Dialogisme », in Détrie C., Siblot P., Verine B. et Steuckardt A. (éds), Termes et concepts pour l'analyse du discours. Une approche praxématique, Paris, H. Champion.

BRES Jacques, 2016, « De la notion d'énoncé dialogique », in Colas-Blaise M., Perrin L. et Tore G.-M. (éds), L'énonciation aujourd'hui. Un concept clé des sciences du langage, Limoges, Lambert Lucas, 119-133.

BRES Jacques, 2001, « Dialogisme (marqueurs de) », in Détrie C., Siblot P. et Verine B. (éds), Termes et concepts pour l'analyse du discours. Une approche praxématique, Paris, H. Champion.

DEBORD Guy, 1967/1992, La société du spectacle, Paris, Gallimard.

DEBORD Guy, wolman Gil, 1956, « Mode d'emploi du détournement », Les lèvres nues, 8.

DEVRIENDT Émilie, à paraître, «La révolution par elle-même : subjectivité radicale et discours de l'action collective dans les inscriptions graffitées pendant l'occupation de la Sorbonne en mai 1968 », in Les acteurs du discours : de l'énonciateur à l'acteur social, Limoges, Lambert Lucas.

DUCARD Dominique, 2015, «Dixit, dicitur, dictum. À propos des Phrases sans texte », in Angermüller J. et Philippe G. (éds), Analyse du discours et dispositifs d'énonciation. Autour des travaux de Dominique Maingueneau, Limoges, Lambert Lucas, 51-59.

GRÉSILLON Almuth, MAINGUENEAU Dominique, 1984, « Polyphonie, proverbe et détournement, ou un proverbe peut en cacher un autre », Langages, 73, 112-125.

LEROY Sarah, 2005, « Le détournement dans les titres de presse : un marquage dialogique?», in Bres J., Haillet P., Mellet S., Nolke H. et Rosier L. (éds), Dialogisme et polyphonie. Approches linguistiques, Bruxelles, De Boeck-Duculot, 201-214.

MAINGUENEAU Dominique, 2012, Les phrases sans texte, Paris, Armand Colin.

MAINGUENEAU Dominique, 2004, « Hyperénonciateur et “particitation” », Langages, 156, 111-126.

MOIRAND Sophie, 2007, « Discours, mémoires et contextes : à propos du fonctionnement de l'allusion dans la presse », Corela, HS-6, URL : https://journals.openedition.org/corela/1567

MOIRAND Sophie, 2004, « Le dialogisme, entre problématiques énonciatives et théories discursives ", Cahiers de praxématique, 43, URL : http://praxematique.revues.org/1853

NO COPYRIGHT, 1998, Sorbonne 68 graffiti, PAGÈs Yves (éd.), Paris, Verticales. 
RABATEL Alain, 2016, « Diversité des points de vue et mobilité empathique », in Colas-Blaise M., Perrin L. et Tore G.-M. (éds), L'énonciation aujourd'hui. Un concept clé des sciences du langage, Limoges, Lambert Lucas, 135-150.

RABATEL Alain, 2015b, « Points de vue en confrontation dans les contrepèteries », in WinterFroemel E. et Zirker A. (éds), Enjeux du jeu des mots. Perspectives linguistiques et littéraires, Amsterdam, Berlin, De Gruyter, Mouton, 31-64.

RABATEL Alain, 2015a, « Postures énonciatives, variables génériques et stratégies de positionnement », in Angermüller J. et Philippe G. (éds), Analyse du discours et dispositifs d'énonciation. Autour des travaux de Dominique Maingueneau, Limoges, Lambert Lucas, 125-135. RABATEL Alain, 2014, « Analyse pragma-énonciative des points de vue en confrontation dans les hyperboles vives : hyper-assertion et sur-énonciation ", in L'hyperbole rhétorique, A. Horak (éd), Travaux neuchâtelois de linguistique, 61-62, 91-109.

RABATEL Alain, 2013, « Humour et sous-énonciation (vs ironie et sur-énonciation) ", L'information grammaticale, 137, 36-42.

RABATEL Alain, 2012b, « Ironie et sur-énonciation », Vox Romanica, 71, 42-76.

RABATEL Alain, 2012a, « Positions, positionnements et postures de l'énonciateur ", Travaux neuchâtelois de linguistique, 56, 23-42.

RABATEL Alain, 2008b, « Figures et points de vue en confrontation », Langue française, 160, 3-17.

RABATEL Alain, 2008a, « Points de vue en confrontation dans les antimétaboles PLUS et MOINS », Langue française, 160, 21-36.

RABATEL Alain, 2004, «L'effacement énonciatif dans les discours rapportés et ses effets pragmatiques », Langages, 156, 3-17.

SALVAN Geneviève, 2010, « Réécrire de connivence : les fortunes dialogiques de l'allusion », Ci-Dit, Communications du IVe Ci-dit, URL : http://revel.unice.fr/symposia/cidit/index.html?id=604 SIBALIS Michael, 2013, « Mai 68 : le Comité d'Action Pédérastique Révolutionnaire occupe la Sorbonne ", Genre, sexualité \& société, 10, URL : http://journals.openedition.org/gss/3009 SITRI Frédérique, 2003, L'objet du débat : la construction des objets de discours dans des situations argumentatives orales, Paris, Presses de la Sorbonne Nouvelle.

VIÉNET René, 1968, Enragés et situationnistes dans le mouvement des occupations, Paris, Gallimard.

\section{NOTES}

1. Les actes sont à paraître chez Lambert Lucas en 2019. Voir Devriendt (à paraître).

2. Transcrites par cinq technicien'ne's d'un laboratoire de psychologie sociale associé au CNRS, publiées depuis (No Copyright 1998). À mon corpus s'ajoutent désormais les 355 inscriptions copiées au centre Censier, publiées ibid.

3. Dans le sous-corpus Sorbonne, l'estimation la plus basse (correspondant aux détournements effectivement identifiés) correspond à $40 \%$ des graffitis (contre $10 \%$ à Censier). Sur l'identification des détournements, voir la section 1.2.

4. Bien qu'aucun de ces travaux ne cite, à ma connaissance, l'origine de l'appellation qu'ils utilisent, celle-ci me semble pouvoir être rattachée à l'usage de ce mot-concept chez les Situationnistes : voir Debord et Wolman (1956), et Debord (1967/1992 : 197), où 
le détournement est défini, avec le "renversement ", comme un élément du « style insurrectionnel » de la critique du capitalisme. Cette référence n'est pas étrangère à mon corpus - au vu notamment du rôle joué par les Situationnistes et leurs épigones dans l'occupation et le graffitage de la Sorbonne en mai 1968 -, et c'est pourquoi j'ai conservé cette appellation sans l'interroger ici plus avant, et sans exploiter son rapport au contexte mentionné.

5. Sa définition s'appuie sur celle de Grésillon et Maingueneau (1984:114) dans leur étude sur les proverbes, en l'élargissant pour l'appliquer à des énoncés dépourvus de " marques linguistiques de l'énonciation proverbiale ». Ce faisant, elle semble aussi en restreindre l'application aux seuls détournements « de niveau 2 » défini par ces auteures (art.cit. : 115) : ceux qui détournent un énoncé attesté $\mathrm{E}_{0}$ en en modifiant le signifiant - le « niveau 1 » portant sur les « conditions génériques de l'énonciation » et pastichant donc le genre assignable à l'énoncé. Je reviens sur cette étude dans la partie 2.

6. À savoir l'énoncé détourné par $\mathrm{E}_{1}$. Ces notations $\left(\mathrm{E}_{1}, \mathrm{E}_{0}\right)$ sont reprises à Grésillon et Maingueneau (1984) dans la mesure où je me réfère souvent à cette étude par la suite. Notons que $\mathrm{E}_{1}$ (qui correspond à (E) chez Bres) ne doit pas être confondu avec E1, l'énonciateur premier dans la notation de Rabatel, souvent en syncrétisme avec L1 (cf la notation L1/E1).

7. Le code précédant les énoncés doit être lu comme suit : la lettre désigne le lieu d'attestation de l'inscription (C pour Censier, S pour Sorbonne), les chiffres correspondent à l'ordre d'apparition de l'inscription dans l'édition Verticales. La graphie de la transcription a été respectée, et les barres obliques notent les passages à la ligne.

8. La conception du dialogisme chez Bres a évolué (voir Bres 2016 : 125 sq., 131) et je mentionnerai donc ici ses propositions plus récentes concernant le détournement.

9. Pour relativiser cette dernière affirmation, voir Leroy (2005:207). Dans ma partie 3 , j'étudierai des cas relevant du dialogisme interlocutif (ou interscripturaire).

10. Ces notations correspondent respectivement à l'énonciateur citant et à l'énonciateur cité.

11. De ce point de vue, bien que les titres de presse analysés par Leroy soient des « aphorisations » (énoncés originellement autonomes, ou détachés d'un texte), il convient de rappeler qu'elles les étudie dans des « séquences formées du titre proprement dit (...) et du "chapeau" de quelques lignes qui, lui, résume l'article » (art. cit. : 202). Mon corpus permet parfois de « textualiser » les inscriptions (voir 3.3), mais ce n'est pas son régime discursif courant : l'identification des détournements s'y avère d'autant plus délicate. Voir aussi note 40 .

12. Rabatel, comme Bres, s'oppose à la conception d'un sujet «plein » (Rabatel $2004: 7$; 2015a : 125, 132), en appui sur Authier-Revuz (1995). Leurs positions diffèrent en revanche sur la question de l'intentionnalité, réintroduite sous certaines conditions par Rabatel (voir 2015a).

13. Voir par exemple Rabatel $(2012,2013,2014)$.

14. Dans Rabatel (2015a), il précise que « (...) les postures renvoient au fait que l'intrication des contenus, aux plans représentationnel et argumentatif, est plus qu'un phénomène polyphonique (entrelacement des voix) ou dialogique (entrelacement des PDV) : un jeu interactionnel de positionnement par rapport aux autres, avec les profits qui lui sont associés. D'où l'articulation des postures avec le positionnement 
sociologique... » (p. 128). Sur cette articulation et plus généralement le rapport des postures au social, voir aussi Rabatel $(2004,2016)$. J'y reviens en 3.3 .

15. Rappelons que mon étude ne porte pas que sur les détournements proverbiaux, d'ailleurs peu nombreux dans mon corpus.

16. Comme le précisent les auteures (p. 114 et 119), une telle dichotomisation doit être relativisée. De fait, bon nombre de détournements dans mon corpus relèvent d'une énonciation ludique à assertion sérieuse.

17. Ce qui implique, comme on le verra plus loin, qu'il puisse y avoir des cas où la captation (pragmatique) est dissociée de l'accord (sémantique), et où la subversion (pragmatique) est dissociée du désaccord (niveau sémantique).

18. Je reprends la notation et la définition proposées, par exemple, dans Rabatel (2014: 92) : « Le PDV correspond (...) aux choix lexicaux, morpho-syntaxiques dans la référenciation d'un contenu propositionnel, car ces derniers expriment le point de vue de l'énonciateur sur l'objet dénoté. »

19. E1' note un énonciateur primaire « autre de soi ».

20. Dans Rabatel (2008b), ces cas sont traités sans recourir aux postures énonciatives. Dans Rabatel (2012a : 38), il laisse toutefois la porte ouverte à cette possibilité. Voir aussi sa mise au point à l'issue du colloque de Toulon. Je n'aborderai pas de front cette question.

21. Grésillon et Maingueneau (art. cit. : 115, note 10) parlent de « parasitisme » à propos de la captation.

22. Ce qui semble limiter la portée de cette approche au vu de la nature anti-autoritaire (ou « contestataire ») de mon corpus. Voir toutefois note 20.

23. Il s'agit d'un " mot-valise qui mêle "participation" et "citation" » Maingueneau (2004 : 112) - pour définir un « régime citationnel » particulier - donc une « catégorie foncièrement pragmatique ». Dialoguant avec Maingueneau (2004:113), Rabatel

(2015a : 128-129) associe quant à lui « particitations » (sentencieuses) et surénonciation, et rappelle par ailleurs la variabilité des postures. Cela étant, je ne suis pas sûre que leurs définitions de l'« hyperénonciateur » se recoupent parfaitement.

24. L'énoncé initial est aussi connu sous la forme Prolétaires de tous les pays, unissez-vous. A priori, Travailleurs est ici une simple variante qui ne doit rien au détournement.

25. Le nom enragé peut désigner soit un groupe politique proche des Situationnistes (Les enragés, avec ou sans majuscule), très actif dans le mouvement des occupations particulièrement à la Sorbonne, où il est " sur-scripteur ", après l'avoir été à Nanterre -, soit les révolutionnaires dans leur ensemble. En auto-désignation, l'emploi est toujours positif.

26. S-386 « On crèvera / les organisateurs / de l'ennui ».

27. Cette analyse reste discutable, dans la mesure où on peut imaginer des graffiteurs cartésiens, qui n'adhèreraient pas forcément, avec Marx, à l'idée que ce n'est pas la conscience qui détermine la vie mais la vie qui détermine la conscience.

28. Si l'on suit Leroy (2005: 207), il s'agit là d'une autre manière de caractériser le dialogisme dit interdiscursif. Voir aussi, par exemple, Moirand (2004).

29. Un exemple prototypique de reprise à l'identique serait C-40 «Fais attention à tes oreilles / elles ont des murs ", qui détourne « Les murs ont des oreilles. Vos oreilles ont des murs » (par ailleurs analysé dans Rabatel 2008b : 25-26).

30. On voit que cette substitution est " significative » s'agissant du $\mathrm{CP}$, contrairement à la substitution homophone de S-84, qui ne joue qu'au niveau référentiel. 
31. Voir aussi Leroy (2005 : 207) : «(...) les énoncés détournés constituent bien des discours antérieurs, des paroles d'autrui, mais, (...) il ne s'agit pas forcément de paroles d'autrui sur le sujet. (...) Le rapport, plus ou moins lointain selon les cas, étant tout au plus secondaire, latéral, on ne parlera pas de dialogisme interdiscursif au sens où $\left[\mathrm{E}_{0}\right]$ serait un discours autre sur l'objet du discours. »

32. Cette pratique correspondrait à la définition du détournement chez Debord (1967 : thèse 208) : «Le détournement est le contraire de la citation, de l'autorité théorique toujours falsifiée du seul fait qu'elle est devenue citation... ». Voir aussi Ducard (2015 : 58).

33. J'entendrai par là ce que Grésillon et Maingueneau (art. cit : 115) nomment " conditions génériques », à savoir : « (...) la réunion des conditions formelles (syntaxiques, prosodiques...) (...) et de certains traits pragmatiques, les conditions d'emploi.»

34. Rabatel (2013) analyse (comme cas de sousénonciation humoristique) un autre détournement de $\mathrm{E}_{0}$, issu du recueil surréaliste de détournements proverbiaux étudié dans Grésillon et Maingueneau (1984) : « Je suis venu, je me suis assis, je suis reparti ». 35. Les inscriptions de la Sorbonne ont été copiées entre les 26 et 29 mai 1968, soit après la seconde nuit des barricades, dont on dit souvent qu'elle a amorcé le reflux du mouvement.

36. Les détournements situationnels (cf partie 1), ou " particitations ", sont également (et principalement) à envisager sous cet angle. Voir par exemple l'inscription d'un vers de Baudelaire pour répondre à une question posée sur un mur (C-229 J'AI ENVIE / DE / BAISER // TOI AUSSI ? ; C-230 ô TOI QUI / le SAVAIS), ou celle d'un vers de Racine pour décrire la situation présente (C-207 « Puisque j’ai commencé de / rompre le silence, / Mâdame, il faut poursuivre. »).

37. Ce fonctionnement peut être comparé à la logique substitutive (vs cumulative) analysée dans Rabatel (2015b) s'agissant des PDV en confrontation dans certaines contrepèteries. Toutes choses égales par ailleurs, on peut aussi, à tout le moins au niveau pragmatique, rapprocher ce fonctionnement des formes (textualisantes) parodiques, où le texte parodié n'est souvent qu'un prétexte à la contestation d'une tout autre cible (voir Aron 2009).

38. Qu'on imagine, par exemple, le détournement Je suis venu, je me suis assis, je suis reparti graffité dans un lieu occupé en mai 68 (université ou usine) : le « farniente » qu'y voit Rabatel $(2013: 38)$ pourrait être interprété comme une pratique beaucoup plus sérieuse, renvoyant à un mot d'ordre révolutionnaire, le « droit à la paresse » envisagé dans la perspective de l'abolition du rapport salarial capitaliste (Voir sa formulation situationniste en S-209 : « NE TRAVAILLEZ / JAMAIS ! / VIVEZ ! »). Dès lors, on aurait à tout le moins un cas de surénonciation.

39. Faute de place je ne traiterai ici que des interactions graffitées interscripturaires. Pour un premier aperçu des interactions intrascripturaires (ex. S-323 « Professeurs, vous nous faites vieillir [et vomir]»), voir Devriendt (à paraître).

40. Sur le rôle des « indices » de la confrontation de PDV (dans l'énonciation non sérieuse des contrepèteries) in praesentia et in absentia, voir Rabatel (2015b : 61-62). 41. Je parle bien ici d'objet aux plans sémantique et propositionnel, et non de l'objet discursif au sens de, par exemple, Sitri (2003) : dans ce dernier sens, l'énonciation textualisante des interactions graffitées implique bien en effet la construction d'un objet commun. 
42. Sur le rôle de l'énonciataire dans l'énonciation dialogique du détournement, voir Leroy (2005), qui reprend Authier-Revuz (2000). Voir aussi Rabatel (2015b : 62) à propos des contrepèteries.

43. Le graffiti situationniste pourrait être lui-même un détournement situationnel (!) d'une phrase d'Anouilh.

44. À titre de comparaison, on peut compléter l'analyse de S-40 (voir 3.1), où le paramètre 3 de la prise en compte peut sembler plus facilement identifiable : le détournement vise 12/e2, le " pape » du Surréalisme - ou tout aphoriseur potentiel de $\mathrm{E}_{0}$ - (soit pour subvertir son autorité, soit pour la capter - par allusion, peut-être, à la « révolution surréaliste »). Reste que, dans tous les cas, on peut y voir le «marquage de territoire » d'un graffiteur possiblement issu du Comité d'Action Pédérastique Révolutionnaire (Sibalis 2013). La substitution du CP pointe de fait avant tout vers le contexte, non seulement par le sujet (la révolution), mais aussi par le prédicat, explicite - y compris du fait de la négation dialogique de la seconde proposition reprise à l'identique (ou ne sera pas) - quant aux enjeux des luttes minoritaires au sein même du mouvement révolutionnaire.

45. Je n'envisage pas ici les cas où $\mathrm{L} 1$ ne repère pas lui-même cette allusion/ réminiscence. Voir Authier-Revuz (1995/2012, 2000), Rabatel (2004 : 16, n. 19).

\section{RÉSUMÉS}

Cet article interroge les conditions d'application des postures énonciatives définies par Rabatel, dans un corpus de "détournements " graffités dans deux enceintes universitaires parisiennes occupées / réappropriées en mai-juin 1968 (Sorbonne, Censier). Dans l'optique de Rabatel, les détournements, en tant qu'énoncés dialogiques, sont analysables comme des phénomènes de points de vue en confrontation, cumulatifs ou substitutifs. Or j'entends montrer qu'une telle analyse ne peut être menée qu'à condition de spécifier en trois paramètres l'objet pris en compte, identifiable comme support de confrontation (ou interaction) dialogique entre l'énoncé initial et son détournement : contenu propositionnel, conditions génériques de l'énonciation, source énonciative de l'énoncé détourné envisagée en tant qu'être du monde. L'analyse du dialogisme des détournements implique donc bien de dissocier, mais aussi de chercher à articuler, les niveaux sémantique, énonciatif, pragmatique et sociodiscursif.

This article aims at questioning the applicability of enunciative postures defined by Rabatel, analyzing a corpus of dialogical utterances (detournements) written on the walls of two occupied/reclaimed faculties in may-june 1968 in Paris, France. Following Rabatel, detournements can be analyzed as confronted points of view. I first illustrate how this analysis works and can be insightful to deal with my corpus, but still addresses issues which make me suggest that the approach of Rabatel can only be applied to such a corpus if one specifies what the object taken into account stands for. I then define the parameters which the dialogical confrontation between the original utterance and its detournement can be based on. The article thus illustrates how the analysis of dialogism in detournements implies to both dissociate and articulate semantical, enunciative, pragmatical and sociodiscursive levels. 
INDEX

Mots-clés : dialogisme, détournement, point de vue, postures énonciatives, discours

révolutionnaires, Mai 68

Keywords : dialogism, detournement, point of view, enunciative postures, revolutionary discourses, May 68

\section{AUTEUR}

\section{ÉMILIE DEVRIENDT}

Laboratoire Babel (EA 2649), Université de Toulon 